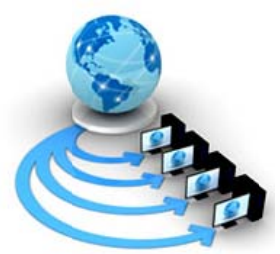

Volume 9, No. 1, January-February 2018

International Journal of Advanced Research in Computer Science

RESEARCH PAPER

\title{
ANALYSIS \& PREVENTION OF POSSIBLE THREATS FOR INTERNET CONNECTED DEVICES IN THE URBAN JUNGLE OF INTERNET OF THINGS
}

\author{
T. Phaniraj Kumar, and V Ramesh \\ Assistant Professor, \\ TKR College of Engineering \& Technology, Hyderabad, India
}

\begin{abstract}
An IoT gadget that plays out a solitary task and does it out in the open will be assaulted in exceptionally distinctive courses from a server that stays securely secured in the server farm, running numerous applications that individuals access from everywhere throughout the world. This article talks about the dangers that are postured by threatening physical access and afterward the dangers that are shared between IoT gadgets and other PCs. As we distinguish and avoid dangers, recollect that security is never static. In security, we need to expect that there is an astute aggressor who is against us who will endeavor to incapacitate or manhandle any security instruments that we set up. A major piece of our security examination is to distinguish the security systems that could help us and how to ensure those instruments from aggressors.
\end{abstract}

Keywords: An IoT gadget that plays out a solitary task and does it out in the open will be assaulted in exceptionally distinctive courses from a server that stays securely secured in the server farm

\section{INTRODUCTION}

Web associated gadgets empower consistent associations among individuals, systems, and physical administrations. These associations manage the cost of efficiencies, novel uses, and altered encounters that are alluring to the two makers and customers. System associated gadgets are as of now getting to be plainly pervasive in, and even basic to, numerous parts of everyday life, from wellness trackers, pacemakers, and autos, to the control frameworks that convey water and energy to our homes. The guarantee offered by IoT nearly unbounded. It is basic that legislature and industry cooperate, rapidly, to guarantee the IoT biological system is based on an establishment that is reliable and secure. In 2014, the President's National Security Telecommunications Advisory Committee (NSTAC) featured the requirement for dire activity. IoT reception will increment in both speed and scope, and will affect basically all segments of our general public. The Nation's test is guaranteeing that the IoT's reception does not make undue risk. Additionally there is a little and quickly shutting window to guarantee that IoT is embraced in a way that expands security and limits chance. On the off chance that the nation neglects to do as such, it will adapt to the outcomes for ages. An opportunity to address IoT security is correct now.It is an initial step to spur and edge discussions about positive measures for IoT security among IoT engineers, producers, specialist organizations, and the clients who buy and convey the gadgets, services, and systems.

The following standards and proposed rehearses give a key concentrate on security and upgrade the trust structure that supports the IoT environment.

\section{Prioritizing IOT Security:}

While the advantages of IoT are evident, actually security isn't staying aware of the pace of development. As we progressively integrate network associations into our country's basic foundation, vital procedures that used to be performed physically (and in this manner appreciated a measure of invulnerability against noxious digital movement) are presently powerless against digital dangers. Our expanding national reliance on arrange - associated advancements has become quicker than the way to secure it. The IoT biological system presents chances that incorporate vindictive on-screen characters controlling the stream of data to and from organize associated gadgets or messing with gadgets themselves, which can prompt the burglary of touchy information and loss of customer protection, interference of business operations, log jam of web usefulness through extensive scale conveyed disavowal of-benefit assaults, and potential disturbances to basic framework Last year, in a digital assault that briefly debilitated the power network in parts of Ukraine, the world saw the basic outcomes that can come about because of disappointments in associated frameworks. Since our country is currently reliant on legitimately working systems to drive such a large number of life-supporting exercises, IoT security is presently a matter of country security.

\section{Physical access breach:}

The clearest dangers for IoT gadgets are physical ones. In many applications, some person could come in, snatch the gadget, and later supplant it with an "enhanced" adaptation that they control. Unless we can control the gadget's area and the general population who can get to it, this hazard is unavoidable. What we can do, however, is recognize when 
the gadget is killed or opened, which are as a rule required to figure out and "include highlights" to it.

\section{Configuration and authentication level breach:}

When a new device is activated and contacts the server for the first time, we need to verify that it is indeed one of our devices and not a hacker's computer. One security measure is to configure a shared secret before we ship the device (a different shared secret for each device). We might consider other security measures that might be easier if we are producing a large number of devices.

\section{Ping/Pulse Message:}

On the off chance that a gadget reports "Hello there, I'm as yet alive" at regular intervals, it is hard to turn it off for over five seconds without we thinking about it. Obviously, in the event that it sends a similar message at regular intervals, it is inconsequential to parody. To make a pulse message that is hard to parody (before we take control of the gadget, in light of the fact that after we take control of a gadget, anything the gadget can do we can do as well), we can send a string with the time (so it will be another string unfailingly), trailed by a cryptographic hash capacity of the time, alongside a common mystery. We can see a well ordered outline of this procedure in Figure 1.

Using Hash Function \& Cryptographic Principals to generate Unique \& Secure Ping/Pulse Message.
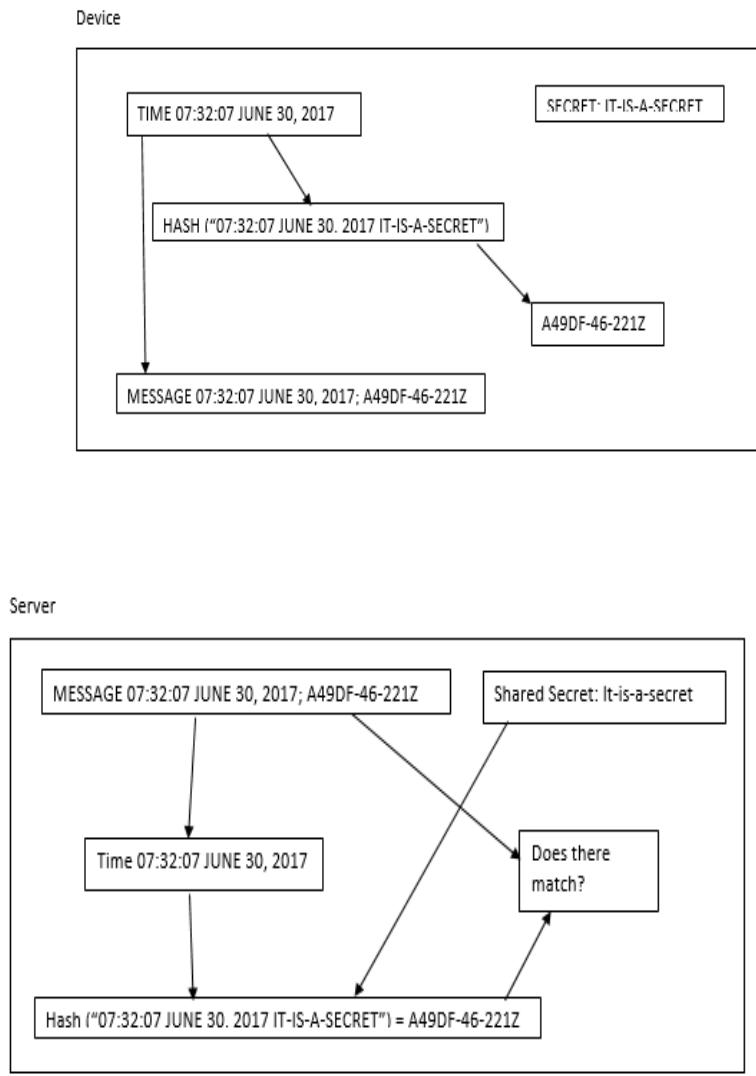

\section{Figure 1}

For such applications, creating false negatives would be troublesome. The arithmetic behind cryptographic hash ${ }^{[2]}$ capacities is sound. Be that as it may, false positives are unimportant. Every one of the assailant needs to do is kill the gadget or separate it from the system. We can make these assaults harder by utilizing USB batteries or reinforcement systems or reinforcement gadgets.

\section{CONNECTIVITY ISSUES/THREATS:}

Most IoT gadgets utilize a buyer review wifi organize. Those systems are quick, modest, and work more often than not. However, wifi arranges regularly have a great deal of real blackouts, which can make following the pulse of IoT gadgets troublesome. Think about these two conceivable answers for this issue:

Get a reinforcement organize, for example, a cell arrange, to guarantee arrange availability. Albeit cell systems have a tendency to be slower, they do have less blackouts. On the off chance that we have the financial plan, this arrangement accommodates solid system availability.

Have an adjacent gadget in a safe area (in a bolted room) get the pulse. At the point when the one gadget winds up noticeably inaccessible, the auxiliary gadget turns into the entrance point and keeps on getting the pulse. At the point when availability is continued, it can answer to our server that the pulse proceeded with authentically. This arrangement is likely much cheaper.The issue with utilizing an optional gadget for pulse checking is that an assailant could separate the system, assume control the two gadgets, and afterward have the auxiliary gadget imagine that the pulse has never been intruded. The answer for this issue is an"auto-lobotomy." If the two gadgets have batteries, it is hard to cripple them two at the very same time. When one gadget recognizes that the other is incapacitated, it can erase the mutual mystery that it utilizes for its own particular pulse. At that point, regardless of whether it is assumed control, it can't be utilized to make counterfeit messages. In any case, erasing the mutual mystery won't not be as straightforward as erasing the record (on the grounds that our document can without much of a stretch be recouped). In the event that there is on-chip stockpiling, utilizing it is most likely the best place to store the common mystery. In the event that there isn't on-chip stockpiling, for instance in a Raspberry $\mathrm{Pi}$, it may be a smart thought to compose however much irregular information into the document framework as could reasonably be expected after we erase the key.

\section{TAMPER PROOF PHYSICAL CASING:}

IoT gadgets are commonly set in boxes to shield them from clean and dampness. we can recognize if such a container is opened by having electrical contacts on the two sides of the cover. On the off chance that the cover is opened, the contacts are disengaged from each other and that is noticeable. Lamentably, this trap is anything but difficult to go around. Jab gaps in the crate, short the associations with each other, and after that opening the case will be imperceptible. Figure 2 for a representation of this alter apparent physical plan for IoT gadget. 


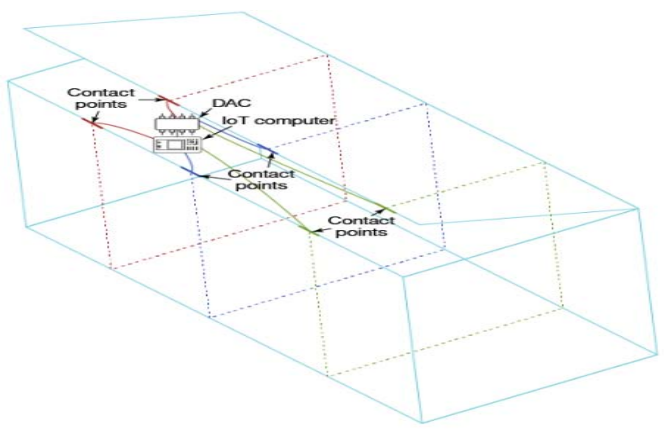

Fig 2

False Inputs from Data Gathering Field Sensors: Physical access takes into consideration another assault on IoT gadgets. An assailant can give sensors false data. For instance, a light sensor can be utilized amid sunlight hours to get data about the climate. However, in the event that someone focuses an electric lamp at the sensor, or puts hazy tape over it, at that point that data will be false.One conceivable arrangement is to include once-overs to make sure everything seems ok for the qualities that originate from our sensors. On the off chance that a sunshine sensor recognizes light during the evening or doesn't distinguish light amid the day, ourIoT gadgets have likely been traded off. On the off chance that it recognizes a similar light level at day break, twelve, and nightfall, it is likely that ourIoT gadgets were bargained. A couple of once-overs to verify everything seems ok on the sensor information make counterfeit info that gets acknowledged a considerable measure harder to deliver. In the event that the gadget isn't utilized autonomously, however as a major aspect of a system of sensors, we can likewise look at comes about because of better places. On the off chance that the sun is sparkling in one place, yet it is totally dim a large portion of a mile away, we may associate a rupture with some kind.

\section{ENHANCING SECURITY OF IOT NETWORK:}

An IoT gadget is a PC that is associated with the web. While IoT gadgets contrast from PCs, all that we do to secure a PC we ought to improve the situation an IoT gadget. To pick the real illustrations, we have to guarantee just appropriately secured server forms are available to the web so they won't be utilized to break into the gadget, scramble anything we exchange, and power clients to change the default qualification on the authoritative account. One essential contrast exists between an IoT gadget and a universally useful PC. Broadly useful PCs have an exceptionally adaptable use design. An IoT gadget, regardless of whether it can do as such significantly more, is ordinarily utilized as a part of an extremely confined mold to execute one or a couple of capacities

Open Ports: Some open system ports are important. In the event that a gadget has an electronic interface, it needs to have an open port for the HTTP server ${ }^{[1]}$ (ideally 443, and ideally we should utilize HTTPS). In the event that an IoT gadget utilizes MQTT as a customer (and it likely will, since MQTT is frequently observed as extraordinary compared to other system conventions for IoT arrangements), for instance, at that point that port should be open for an active association. Nonetheless, every association through the system is a potential security chance. The CVE Details site incorporates a rundown of vulnerabilities, which incorporates just distributed vulnerabilities. An obscure number of unpublished vulnerabilities likewise exist, potentially influencing the projects that we use in ourIoT gadgets. While we have to permit some system access for the IoT gadget to carry out its activity, anything past that is a pointless hazard and we ought to deny it. This procedure is the fundamental security guideline known as "Slightest Privilege". The most straightforward approach to piece organize get to when we utilize a universally useful working framework is to utilize a firewall. On the off chance that ourIoT gadget utilizes Linux, we can utilize the IP tables application to guarantee firewall uptime and security.

\section{(i) ENCRYPTION AND DECRYPTION:}

From a system security viewpoint, two conceivable assaults can happen on data while it ventures to every part of the web, regardless of whether from the IoT gadget to a focal server or from that server back to the IoT gadget.

Theft/Spoofing: The IoT data may be profitable for other individuals. For instance, realizing that the cooling has been off for the most recent week, amidst a Texas summer, can tell robbers that a family is in the midst of some recreation, the house is vacant, and the substance of the house can be theirs for the taking.

\section{DISGUISING, ALTERATION, OR ERASURE:}

If the house has an alert framework that can be remotely controlled, the thieves would be significantly more joyful on the off chance that they could send an incapacitate charge before they soften up.One Possible arrangement with the business standard arrangement, TLS, is the manin-the-center assault. TLS incorporates an answer, testaments that recognize servers, yet those endorsements just secure we on the off chance that we really confirm the personality that is encoded in them.

Securing Credentials: IoT gadgets are ordinarily directed remotely, through the system. More often than not, when the gadget originates from the industrial facility it utilized default certifications (client name and secret word), which clients should change. On the off chance that the client does not change the default accreditations, aggressors can utilize them to control the gadget. This assault has just happened to IoT gadgets - the Mirari, BrikerBot, and Amnesia malware programs have utilized default accreditations to break into IoT gadgets. To maintain a strategic distance from this issue, expect clients to change the default secret key when they sign on out of the blue, before they can do whatever else. 


\section{CONCLUSION}

We can utilize this abnormal state diagram of the security dangers against IoT gadgets to consider the dangers against we own gadgets and how to alleviate them. Culminate security is inconceivable, however it is conceivable to make weIoT gadgets harder to assault than different targets.

\section{BIBLIOGRAPHY:}

[1] Secure Communication Algorithm in Web-Services by Mamidala Naveen Kumar, Shaik Khaja Hafeezuddin, G Kumar. IJSR Volume 5 Issue 11.
[2] F. Guo, Y. Mu, Z. Chen, and L. Xu, "Multi-Identity Single-Key Decryption without Random Oracles,” in Proceedings of Information Security and Cryptology (Inscrypt ’07), ser. LNCS, vol. 4990. Springer, 2007, pp. 384-398.

[3] W.G. Tzeng, "A Time-Bound Cryptographic Key Assignment Scheme for Access Control in a Hierarchy," IEEE Transactions on Knowledge and Data Engineering (TKDE), vol. 14, no. 1, pp. 182- 188,2002site).

[4] D. Boneh and M. K. Franklin, "Identity-Based Encryption from the Weil Pairing," in Proceedings of advances in cryptology - CRYPTO '01, ser.LNCS, vol.2139. Springer, 2001, pp. 213-229 